\title{
Complete moment convergence for randomly weighted sums of martingale differences
}

\author{
Wenzhi Yang ${ }^{1}$, Yiwei Wang ${ }^{2}$, Xinghui Wang ${ }^{1}$ and Shuhe $\mathrm{Hu}^{1 *}$
}

\section{"Correspondence:}

hushuhe@263.net

'School of Mathematical Science,

Anhui University, Hefei, China

Full list of author information is

available at the end of the article

\begin{abstract}
In this article, we obtain the complete moment convergence for randomly weighted sums of martingale differences. Our results generalize the corresponding ones for the nonweighted sums of martingale differences to the case of randomly weighted sums of martingale differences.
\end{abstract}

MSC: $60 \mathrm{G} 50 ; 60 \mathrm{~F} 15$

Keywords: complete convergence; randomly weighted sums; martingale differences

\section{Introduction}

The concept of complete convergence was introduced by Hsu and Robbins [1], i.e., a sequence of random variables $\left\{X_{n}, n \geq 1\right\}$ is said to converge completely to a constant $C$ if $\sum_{n=1}^{\infty} P\left(\left|X_{n}-C\right| \geq \varepsilon\right)<\infty$ for all $\varepsilon>0$. In view of Borel-Cantelli lemma, this implies that $X_{n} \rightarrow C$ almost surely (a.s.). The converse is true if $\left\{X_{n}, n \geq 1\right\}$ is independent. Hsu and Robbins [1] obtained that the sequence of arithmetic means of independent and identically distributed (i.i.d.) random variables converges completely to the expected value if the variance of the summands is finite. Erdös [2] proved the converse. The result of HsuRobbins-Erdös is a fundamental theorem in probability theory, and it has been generalized and extended in several directions by many authors. Baum and Katz [3] gave the following generalization to establish a rate of convergence in the sense of Marcinkiewicz-Zygmundtype strong law of large numbers.

Theorem 1.1 Let $\alpha>1 / 2, \alpha p>1$ and $\left\{X_{n}, n \geq 1\right\}$ be a sequence of i.i.d. random variables. Assume that $E X_{1}=0$ if $\alpha \leq 1$. Then the following statements are equivalent

(i) $E\left|X_{1}\right|^{p}<\infty$;

(ii) $\sum_{n=1}^{\infty} n^{\alpha p-2} P\left(\max _{1 \leq k \leq n}\left|\sum_{i=1}^{k} X_{i}\right|>\varepsilon n^{\alpha}\right)<\infty$ for all $\varepsilon>0$.

Many authors have extended Theorem 1.1 for the i.i.d. case to some dependent cases. For example, Shao [4] investigated the moment inequalities for the $\varphi$-mixing random variables and gave its application to the complete convergence for this stochastic process; $\mathrm{Yu}$ [5] obtained the complete convergence for weighted sums of martingale differences; Ghosal and Chandra [6] gave the complete convergence of martingale arrays; Stoica [7, 8] investigated the Baum-Katz-Nagaev-type results for martingale differences and the rate of convergence 
in the strong law of large numbers for martingale differences; Wang et al. [9] also studied the complete convergence and complete moment convergence for martingale differences, which generalized some results of Stoica [7, 8]; Yang et al. [10] obtained the complete convergence for the moving average process of martingale differences and so forth. For other works about convergence analysis, one can refer to Gut [11], Chen et al. [12], Sung [13-16], Sung and Volodin [17], Hu et al. [18] and the references therein.

Recently, Thanh and Yin [19] studied the complete convergence for randomly weighted sums of independent random elements in Banach spaces. On the other hand, Cabrera et al. [20] investigated some theorems on conditional mean convergence and conditional almost sure convergence for randomly weighted sums of dependent random variables. Inspired by the papers above, we will investigate the complete moment convergence for randomly weighted sums of martingale differences in this paper, which implies the complete convergence and Marcinkiewicz-Zygmund-type strong law of large numbers for this stochastic process. We generalize the results of Stoica [7, 8] and Wang et al. [9] for the nonweighted sums of martingale differences to the case of randomly weighted sums of martingale differences. For the details, one can refer to the main results presented in Section 2. The proofs of the main results are presented in Section 3.

Recall that the sequence $\left\{X_{n}, n \geq 1\right\}$ is stochastically dominated by a nonnegative random variable $X$ if

$$
\sup _{n \geq 1} P\left(\left|X_{n}\right|>t\right) \leq C P(X>t) \quad \text { for some positive constant } C \text { and for all } t \geq 0 .
$$

Throughout the paper, let $\mathcal{F}_{0}=\{\emptyset, \Omega\}, x^{+}=x I(x \geq 0), I(B)$ be the indicator function of set $B$ and $C, C_{1}, C_{2}, \ldots$ denote some positive constants not depending on $n$, which may be different in various places.

To prove the main results of the paper, we need the following lemmas.

Lemma 1.1 (cf. Hall and Heyde [21], Theorem 2.11) If $\left\{X_{i}, \mathcal{F}_{i}, 1 \leq i \leq n\right\}$ is a martingale difference and $p>0$, then there exists a constant $C$ depending only on $p$ such that

$$
E\left(\max _{1 \leq k \leq n}\left|\sum_{i=1}^{k} X_{i}\right|^{p}\right) \leq C\left\{E\left(\sum_{i=1}^{n} E\left(X_{i}^{2} \mid \mathcal{F}_{i-1}\right)\right)^{p / 2}+E\left(\max _{1 \leq i \leq n}\left|X_{i}\right|^{p}\right)\right\}, \quad n \geq 1 .
$$

Lemma 1.2 (cf. Sung [13], Lemma 2.4) Let $\left\{X_{n}, n \geq 1\right\}$ and $\left\{Y_{n}, n \geq 1\right\}$ be sequences of random variables. Then for any $n \geq 1, q>1, \varepsilon>0$ and $a>0$,

$$
\begin{aligned}
E\left(\max _{1 \leq j \leq n}\left|\sum_{i=1}^{j}\left(X_{i}+Y_{i}\right)\right|-\varepsilon a\right)^{+} \leq & \left(\frac{1}{\varepsilon^{q}}+\frac{1}{q-1}\right) \frac{1}{a^{q-1}} E\left(\max _{1 \leq j \leq n}\left|\sum_{i=1}^{j} X_{i}\right|^{q}\right) \\
& +E\left(\max _{1 \leq j \leq n}\left|\sum_{i=1}^{j} Y_{i}\right|\right) .
\end{aligned}
$$

Lemma 1.3 (cf. Wang et al. [9], Lemma 2.2) Let $\left\{X_{n}, n \geq 1\right\}$ be a sequence of random variables stochastically dominated by a nonnegative random variable $X$. Then for any $n \geq 1$, 
$a>0$ and $b>0$, the following two statements hold

$$
E\left[\left|X_{n}\right|^{a} I\left(\left|X_{n}\right| \leq b\right)\right] \leq C_{1}\left\{E\left[X^{a} I(X \leq b)\right]+b^{a} P(X>b)\right\}
$$

and

$$
E\left[\left|X_{n}\right|^{a} I\left(\left|X_{n}\right|>b\right)\right] \leq C_{2} E\left[X^{a} I(X>b)\right]
$$

where $C_{1}$ and $C_{2}$ are positive constants.

\section{Main results}

Theorem 2.1 Let $\alpha>1 / 2,1<p<2,1 \leq \alpha p<2$ and $\left\{X_{n}, \mathcal{F}_{n}, n \geq 1\right\}$ be a martingale difference sequence stochastically dominated by a nonnegative random variable $X$ with $E X^{p}<\infty$. Assume that $\left\{A_{n}, n \geq 1\right\}$ is a random sequence, and it is independent of $\left\{X_{n}, n \geq 1\right\}$. If

$$
\sum_{i=1}^{n} E A_{i}^{2}=O(n)
$$

then for every $\varepsilon>0$,

$$
\sum_{n=1}^{\infty} n^{\alpha p-2-\alpha} E\left(\max _{1 \leq k \leq n}\left|\sum_{i=1}^{k} A_{i} X_{i}\right|-\varepsilon n^{\alpha}\right)^{+}<\infty
$$

and for $\alpha p>1$,

$$
\sum_{n=1}^{\infty} n^{\alpha p-2} E\left(\sup _{k \geq n}\left|\frac{\sum_{i=1}^{k} A_{i} X_{i}}{k^{\alpha}}\right|-\varepsilon\right)^{+}<\infty
$$

Theorem 2.2 Let $\alpha>1 / 2, p \geq 2$ and $\left\{X_{n}, \mathcal{F}_{n}, n \geq 1\right\}$ be a martingale difference sequence stochastically dominated by a nonnegative random variable $X$ with $E X^{p}<\infty$. Let $\left\{A_{n}, n \geq\right.$ $1\}$ be a random sequence, which is independent of $\left\{X_{n}, n \geq 1\right\}$. Denote $g_{0}=\{\emptyset, \Omega\}$ and $g_{n}=$ $\sigma\left(X_{1}, \ldots, X_{n}\right), n \geq 1$. For some $q>\frac{2(\alpha p-1)}{2 \alpha-1}$, we assume that $E\left[\sup _{n \geq 1} E\left(X_{n}^{2} \mid g_{n-1}\right)\right]^{q / 2}<\infty$ and

$$
\sum_{i=1}^{n} E\left|A_{i}\right|^{q}=O(n)
$$

Then for every $\varepsilon>0,(2.2)$ and (2.3) hold.

Meanwhile, for the case $p=1$, we have the following theorem.

Theorem 2.3 Let $\alpha>0$ and $\left\{X_{n}, \mathcal{F}_{n}, n \geq 1\right\}$ be a martingale difference sequence stochastically dominated by a nonnegative random variable $X$ with $E[X \ln (1+X)]<\infty$. Assume that (2.1) holds and $\left\{A_{n}, n \geq 1\right\}$ is a random sequence, which is independent of $\left\{X_{n}, n \geq 1\right\}$. Then for every $\varepsilon>0$,

$$
\sum_{n=1}^{\infty} n^{-2} E\left(\max _{1 \leq k \leq n}\left|\sum_{i=1}^{k} A_{i} X_{i}\right|-\varepsilon n^{\alpha}\right)^{+}<\infty
$$


and for $\alpha>1$,

$$
\sum_{n=1}^{\infty} n^{\alpha-2} E\left(\sup _{k \geq n}\left|\frac{\sum_{i=1}^{k} A_{i} X_{i}}{k^{\alpha}}\right|-\varepsilon\right)^{+}<\infty
$$

In particular, for $\alpha>0$, it has

$$
\sum_{n=1}^{\infty} n^{\alpha-2} P\left(\max _{1 \leq k \leq n}\left|\sum_{i=1}^{k} A_{i} X_{i}\right|>\varepsilon n^{\alpha}\right)<\infty
$$

and for $\alpha>1$, it has

$$
\sum_{n=1}^{\infty} n^{\alpha-2} P\left(\sup _{k \geq n}\left|\frac{\sum_{i=1}^{k} A_{i} X_{i}}{k^{\alpha}}\right|>\varepsilon\right)<\infty
$$

On the other hand, for $\alpha \geq 1$ and $E X<\infty$, we have the following theorem.

Theorem 2.4 Let $\alpha \geq 1$ and $\left\{X_{n}, \mathcal{F}_{n}, n \geq 1\right\}$ be a martingale difference sequence stochastically dominated by a nonnegative random variable $X$ with $E X<\infty$. Denote $g_{0}=\{\emptyset, \Omega\}$ and $g_{n}=\sigma\left(X_{1}, \ldots, X_{n}\right), n \geq 1$. Let (2.1) hold, and let $\left\{A_{n}, n \geq 1\right\}$ be a random sequence, which is independent of $\left\{X_{n}, n \geq 1\right\}$. We assume (i) under the case of $\alpha=1$, there exists $a \delta>0$ such that

$$
\lim _{n \rightarrow \infty} \frac{\max _{1 \leq i \leq n} E\left[\left|X_{i}\right|^{1+\delta} \mid g_{i-1}\right]}{n^{\delta}}=0, \quad \text { a.s. }
$$

and (ii) under the case of $\alpha>1$, it has for any $\lambda>0$ that

$$
\lim _{n \rightarrow \infty} \frac{\max _{1 \leq i \leq n} E\left[\left|X_{i}\right| \mid g_{i-1}\right]}{n^{\lambda}}=0, \quad \text { a.s. }
$$

Then for $\alpha \geq 1$ and every $\varepsilon>0$, it has (2.7). In addition, for $\alpha>1$, it has (2.8).

Remark 2.1 If the conditions of Theorem 2.1 or Theorem 2.2 hold, then for every $\varepsilon>0$,

$$
\sum_{n=1}^{\infty} n^{\alpha p-2} P\left(\max _{1 \leq k \leq n}\left|\sum_{i=1}^{k} A_{i} X_{i}\right|>\varepsilon n^{\alpha}\right)<\infty,
$$

and for $\alpha p>1$,

$$
\sum_{n=1}^{\infty} n^{\alpha p-2} P\left(\sup _{k \geq n}\left|\frac{\sum_{i=1}^{k} A_{i} X_{i}}{k^{\alpha}}\right|>\varepsilon\right)<\infty .
$$

In fact, it can be checked that for every $\varepsilon>0$,

$$
\begin{aligned}
& \sum_{n=1}^{\infty} n^{\alpha p-2-\alpha} E\left(\max _{1 \leq k \leq n}\left|\sum_{i=1}^{k} A_{i} X_{i}\right|-\varepsilon n^{\alpha}\right)^{+} \\
& \quad=\sum_{n=1}^{\infty} n^{\alpha p-2-\alpha} \int_{0}^{\infty} P\left(\max _{1 \leq k \leq n}\left|\sum_{i=1}^{k} A_{i} X_{i}\right|-\varepsilon n^{\alpha}>t\right) d t
\end{aligned}
$$




$$
\begin{aligned}
& \geq \sum_{n=1}^{\infty} n^{\alpha p-2-\alpha} \int_{0}^{\varepsilon n^{\alpha}} P\left(\max _{1 \leq k \leq n}\left|\sum_{i=1}^{k} A_{i} X_{i}\right|-\varepsilon n^{\alpha}>t\right) d t \\
& \geq \varepsilon \sum_{n=1}^{\infty} n^{\alpha p-2} P\left(\max _{1 \leq k \leq n}\left|\sum_{i=1}^{k} A_{i} X_{i}\right|>2 \varepsilon n^{\alpha}\right) .
\end{aligned}
$$

So (2.2) implies (2.9).

On the other hand, by the proof of Theorem 12.1 of Gut [11] and the proof of (3.2) in Yang et al. [10], for $\alpha p>1$, it is easy to see that

$$
\sum_{n=1}^{\infty} n^{\alpha p-2} P\left(\sup _{k \geq n}\left|\frac{\sum_{i=1}^{k} A_{i} X_{i}}{k^{\alpha}}\right|>2^{2 \alpha} \varepsilon\right) \leq C_{1} \sum_{n=1}^{\infty} n^{\alpha p-2} P\left(\max _{1 \leq k \leq n}\left|\sum_{i=1}^{k} A_{i} X_{i}\right|>\varepsilon n^{\alpha}\right) .
$$

Thus (2.10) follows from (2.9).

Remark 2.2 In Theorem 2.1, if $\alpha=1 / p$, then for every $\varepsilon>0$, we get by (2.9) that

$$
\sum_{n=1}^{\infty} n^{-1} P\left(\max _{1 \leq k \leq n}\left|\sum_{i=1}^{k} A_{i} X_{i}\right|>\varepsilon n^{1 / p}\right)<\infty .
$$

By using (2.12), one can easily get the Marcinkiewicz-Zygmund-type strong law of large numbers of randomly weighted sums of martingale difference as following

$$
\lim _{n \rightarrow \infty} \frac{1}{n^{1 / p}} \sum_{i=1}^{n} A_{i} X_{i}=0, \quad \text { a.s. }
$$

If $A_{n}=a_{n}$ is non-random (the case of constant weighted), $n \geq 1$, then one can get the results of Theorems 2.1-2.4 for the non-random weighted sums of martingale differences.

Meanwhile, it can be seen that our condition $E\left[\sup _{n \geq 1} E\left(X_{n}^{2} \mid g_{n-1}\right)\right]^{q / 2}<\infty$ in Theorem 2.2 is weaker than the condition $\sup _{n \geq 1} E\left(X_{n}^{2} \mid \mathscr{F}_{n-1}\right) \leq C$, a.s. in Theorem 1.4, Theorem 1.5 and Theorem 1.7 of Wang et al. [9]. In fact, it follows from $g_{n-1} \subseteq \mathcal{F}_{n-1}$ that

$$
E\left(X_{n}^{2} \mid g_{n-1}\right)=E\left[E\left(X_{n}^{2} \mid \mathcal{F}_{n-1}\right) \mid \mathscr{g}_{n-1}\right] \leq E\left[\sup _{n \geq 1} E\left(X_{n}^{2} \mid \mathcal{F}_{n-1}\right) \mid \mathscr{g}_{n-1}\right]
$$

If $\sup _{n \geq 1} E\left(X_{n}^{2} \mid \mathcal{F}_{n-1}\right) \leq C$, a.s., then it has $E\left[\sup _{n \geq 1} E\left(X_{n}^{2} \mid g_{n-1}\right)\right]^{q / 2}<\infty$. For $\alpha \geq 1$ and $E[X \ln (1+X)]<\infty$, Wang et al. [9] obtained the result of (2.7) (see Theorem 1.6 of Wang et al. [9]). Therefore, by Theorems 2.1-2.4 in this paper, we generalize Theorems 1.4-1.7 of Wang et al. [9] for the nonweighted sums of martingale differences to the case of randomly weighted sums of martingale differences.

On the other hand, let the hypothesis that $\left\{A_{n}, n \geq 1\right\}$ is independent of $\left\{X_{n}, n \geq 1\right\}$ be replaced by that $A_{n}$ is $\mathcal{F}_{n-1}$-measurable and $A_{n}$ is independent of $X_{n}$ for each $n \geq 1$ in Theorem 2.1, and the other conditions of Theorem 2.1 hold, one can get (2.2) and (2.3) (the proof is similar to the one of Theorem 2.1). Let $A_{n}$ be $\mathcal{F}_{n-1}$-measurable, $A_{n}$ be independent of $X_{n}$ for each $n \geq 1, E\left[\sup _{n \geq 1} E\left(X_{n}^{2} \mid \mathcal{F}_{n-1}\right)\right]^{q / 2}<\infty$ and other conditions of Theorem 2.2 hold, one can also obtain (2.2) and (2.3). We can obtain some similar results if we only require $A_{n}$ is $\mathcal{F}_{n-1}$-measurable for all $n \geq 1$ (without any independence hypothesis). This case would have many interesting applications (see Huang and Guo [22], Thanh et al. [23] and the references therein). 


\section{The proofs of main results}

Proof of Theorem 2.1 Let $g_{0}=\{\emptyset, \Omega\}$, for $n \geq 1, g_{n}=\sigma\left(X_{1}, \ldots, X_{n}\right)$ and

$$
X_{n i}=X_{i} I\left(\left|X_{i}\right| \leq n^{\alpha}\right), \quad 1 \leq i \leq n .
$$

It can be seen that

$$
A_{i} X_{i}=A_{i} X_{i} I\left(\left|X_{i}\right|>n^{\alpha}\right)+\left[A_{i} X_{n i}-E\left(A_{i} X_{n i} \mid \mathscr{g}_{i-1}\right)\right]+E\left(A_{i} X_{n i} \mid g_{i-1}\right), \quad 1 \leq i \leq n .
$$

So, by Lemma 1.2 with $a=n^{\alpha}$, for $q>1$, one has that

$$
\begin{aligned}
& \sum_{n=1}^{\infty} n^{\alpha p-2-\alpha} E\left(\max _{1 \leq k \leq n}\left|\sum_{i=1}^{k} A_{i} X_{i}\right|-\varepsilon n^{\alpha}\right)^{+} \\
& \leq C_{1} \sum_{n=1}^{\infty} n^{\alpha p-2-q \alpha} E\left(\max _{1 \leq k \leq n}\left|\sum_{i=1}^{k}\left[A_{i} X_{n i}-E\left(A_{i} X_{n i} \mid g_{i-1}\right)\right]\right|^{q}\right) \\
&+\sum_{n=1}^{\infty} n^{\alpha p-2-\alpha} E\left(\max _{1 \leq k \leq n}\left|\sum_{i=1}^{k}\left[A_{i} X_{i} I\left(\left|X_{i}\right|>n^{\alpha}\right)+E\left(A_{i} X_{n i} \mid g_{i-1}\right)\right]\right|\right) \\
& \leq C_{1} \sum_{n=1}^{\infty} n^{\alpha p-2-q \alpha} E\left(\max _{1 \leq k \leq n}\left|\sum_{i=1}^{k}\left[A_{i} X_{n i}-E\left(A_{i} X_{n i} \mid g_{i-1}\right)\right]\right|^{q}\right) \\
&+\sum_{n=1}^{\infty} n^{\alpha p-2-\alpha} E\left(\max _{1 \leq k \leq n}\left|\sum_{i=1}^{k} A_{i} X_{i} I\left(\left|X_{i}\right|>n^{\alpha}\right)\right|\right) \\
&+\sum_{n=1}^{\infty} n^{\alpha p-2-\alpha} E\left(\max _{1 \leq k \leq n}\left|\sum_{i=1}^{k} E\left(A_{i} X_{n i} \mid g_{i-1}\right)\right|\right) \\
&:= H_{1}+H_{2}+H_{3} .
\end{aligned}
$$

Obviously, it follows from Hölder's inequality and (2.1) that

$$
\sum_{i=1}^{n} E\left|A_{i}\right| \leq\left(\sum_{i=1}^{n} E A_{i}^{2}\right)^{1 / 2}\left(\sum_{i=1}^{n} 1\right)^{1 / 2}=O(n) .
$$

By the fact that $\left\{A_{n}, n \geq 1\right\}$ is independent of $\left\{X_{n}, n \geq 1\right\}$, we can check by Markov's inequality, Lemma 1.3, (3.2) and $E X^{p}<\infty(p>1)$ that

$$
\begin{aligned}
H_{2} & \leq \sum_{n=1}^{\infty} n^{\alpha p-2-\alpha} \sum_{i=1}^{n} E\left|A_{i}\right| E\left[\left|X_{i}\right| I\left(\left|X_{i}\right|>n^{\alpha}\right)\right] \\
& \leq \sum_{n=1}^{\infty} n^{\alpha p-1-\alpha} E\left[X I\left(X>n^{\alpha}\right)\right] \\
& =\sum_{n=1}^{\infty} n^{\alpha p-1-\alpha} \sum_{m=n}^{\infty} E\left[X I\left(m^{\alpha}<X \leq(m+1)^{\alpha}\right)\right]
\end{aligned}
$$




$$
\begin{aligned}
& =\sum_{m=1}^{\infty} E\left[X I\left(m^{\alpha}<X \leq(m+1)^{\alpha}\right)\right] \sum_{n=1}^{m} n^{\alpha p-1-\alpha} \\
& \leq C_{2} \sum_{m=1}^{\infty} m^{\alpha p-\alpha} E\left[X I\left(m^{\alpha}<X \leq(m+1)^{\alpha}\right)\right] \\
& \leq C_{3} E X^{p}<\infty
\end{aligned}
$$

On the other hand, one can see that $\left\{X_{n}, g_{n}, n \geq 1\right\}$ is also a martingale difference, since $\left\{X_{n}, \mathcal{F}_{n}, n \geq 1\right\}$ is a martingale difference. Combining with the fact that $\left\{A_{n}, n \geq 1\right\}$ is independent of $\left\{X_{n}, n \geq 1\right\}$, we have that

$$
\begin{aligned}
E\left(A_{n} X_{n} \mid g_{n-1}\right) & =E\left[E\left(A_{n} X_{n} \mid g_{n}\right) \mid g_{n-1}\right]=E\left[X_{n} E\left(A_{n} \mid g_{n}\right) \mid g_{n-1}\right] \\
& =E A_{n} E\left[X_{n} \mid g_{n-1}\right]=0, \quad \text { a.s., } n \geq 1 .
\end{aligned}
$$

Consequently, by the proof of (3.3), it follows that

$$
\begin{aligned}
H_{3} & =\sum_{n=1}^{\infty} n^{\alpha p-2-\alpha} E\left(\max _{1 \leq k \leq n}\left|\sum_{i=1}^{k} E\left[A_{i} X_{i} I\left(\left|X_{i}\right| \leq n^{\alpha}\right) \mid g_{i-1}\right]\right|\right) \\
& =\sum_{n=1}^{\infty} n^{\alpha p-2-\alpha} E\left(\max _{1 \leq k \leq n}\left|\sum_{i=1}^{k} E\left[A_{i} X_{i} I\left(\left|X_{i}\right|>n^{\alpha}\right) \mid g_{i-1}\right]\right|\right) \\
& \leq \sum_{n=1}^{\infty} n^{\alpha p-2-\alpha} \sum_{i=1}^{n} E\left|A_{i}\right| E\left[\left|X_{i}\right| I\left(\left|X_{i}\right|>n^{\alpha}\right)\right] \\
& \leq C_{4} \sum_{n=1}^{\infty} n^{\alpha p-1-\alpha} E\left[X I\left(X>n^{\alpha}\right)\right] \leq C_{5} E X^{p}<\infty .
\end{aligned}
$$

Next, we turn to prove $H_{1}<\infty$. It can be found that for fixed real numbers $a_{1}, \ldots, a_{n}$,

$$
\left\{a_{i} X_{n i}-E\left(a_{i} X_{n i} \mid g_{i-1}\right), g_{i}, 1 \leq i \leq n\right\}
$$

is also a martingale difference. Note that $\left\{A_{1}, A_{2}, \ldots, A_{n}\right\}$ is independent of $\left\{X_{n 1}, X_{n 2}\right.$, $\left.\ldots, X_{n n}\right\}$. So, by Markov's inequality, (2.1), (3.1) with $q=2$, Lemma 1.1 with $p=2$ and Lemma 1.3, we get that

$$
\begin{aligned}
H_{1} & =C_{1} \sum_{n=1}^{\infty} n^{\alpha p-2-2 \alpha} E\left\{E \max _{1 \leq k \leq n} \sum_{i=1}^{k}\left[a_{i} X_{n i}-E\left(a_{i} X_{n i} \mid g_{i-1}\right)\right]^{2} \mid A_{1}=a_{1}, \ldots, A_{n}=a_{n}\right\} \\
& \leq C_{2} E\left(\sum_{i=1}^{n} E\left(a_{i} X_{n i}\right)^{2} \mid A_{1}=a_{1}, \ldots, A_{n}=a_{n}\right) \\
& =C_{2} \sum_{n=1}^{\infty} n^{\alpha p-2-2 \alpha} \sum_{i=1}^{n} E\left(A_{i} X_{n i}\right)^{2}=C_{2} \sum_{n=1}^{\infty} n^{\alpha p-2-2 \alpha} \sum_{i=1}^{n} E A_{i}^{2} E X_{n i}^{2} \\
& \leq C_{3} \sum_{n=1}^{\infty} n^{\alpha p-1-2 \alpha} E\left[X^{2} I\left(X \leq n^{\alpha}\right)\right]+C_{4} \sum_{n=1}^{\infty} n^{\alpha p-1} P\left(X>n^{\alpha}\right) \\
& =: C_{3} H_{11}+C_{4} H_{12} .
\end{aligned}
$$


By the condition $E X^{p}<\infty$ with $p<2$, it follows

$$
\begin{aligned}
H_{11} & =\sum_{n=1}^{\infty} n^{\alpha p-1-2 \alpha} \sum_{i=1}^{n} E\left[X^{2} I\left((i-1)^{\alpha}<X \leq i^{\alpha}\right)\right] \\
& =\sum_{i=1}^{\infty} E\left[X^{2} I\left((i-1)^{\alpha}<X \leq i^{\alpha}\right)\right] \sum_{n=i}^{\infty} n^{\alpha p-1-2 \alpha} \\
& \leq C_{5} \sum_{i=1}^{\infty} E\left[X^{p} X^{2-p} I\left((i-1)^{\alpha}<X \leq i^{\alpha}\right)\right] i^{\alpha p-2 \alpha} \leq C_{6} E X^{p}<\infty .
\end{aligned}
$$

From (3.3), it has

$$
H_{12} \leq \sum_{n=1}^{\infty} n^{\alpha p-1-\alpha} E\left[X I\left(X>n^{\alpha}\right)\right] \leq C E X^{p}<\infty .
$$

Consequently, by (3.1) and (3.3)-(3.7), we obtain (2.2) immediately.

For $\alpha p>1$, we turn to prove (2.3). Denote $S_{k}=\sum_{i=1}^{k} A_{i} X_{i}, k \geq 1$. It can be seen that $\alpha p<2<2+\alpha$. So, similar to the proof of (3.4) in Yang et al. [10], we can check that

$$
\begin{aligned}
\sum_{n=1}^{\infty} n^{\alpha p-2} E\left(\sup _{k \geq n}\left|\frac{S_{k}}{k^{\alpha}}\right|-\varepsilon 2^{2 \alpha}\right)^{+} & \leq C_{1} \sum_{l=1}^{\infty} 2^{l(\alpha p-1-\alpha)} \int_{0}^{\infty} P\left(\max _{1 \leq k \leq 2^{l}}\left|S_{k}\right|>\varepsilon 2^{\alpha(l+1)}+s\right) d s \\
& \leq C_{1} 2^{2+\alpha-\alpha p} \sum_{n=1}^{\infty} n^{\alpha p-2-\alpha} E\left(\max _{1 \leq k \leq n}\left|S_{k}\right|-\varepsilon n^{\alpha}\right)^{+} .
\end{aligned}
$$

Combining with (2.2), we get (2.3) finally.

Proof of Theorem 2.2 To prove Theorem 2.2, we use the same notation as that in the proof of Theorem 2.1. For $p \geq 2$, it is easy to see that $q>2(\alpha p-1) /(2 \alpha-1) \geq 2$. Consequently, for any $1 \leq s \leq 2$, by Hölder's inequality and (2.4), we get

$$
\sum_{i=1}^{n} E\left|A_{i}\right|^{s} \leq\left(\sum_{i=1}^{n} E\left|A_{i}\right|^{q}\right)^{s / q}\left(\sum_{i=1}^{n} 1\right)^{1-s / q}=O(n) .
$$

By (3.1), (3.3) and (3.4), one can find that $H_{2}<\infty$ and $H_{3}<\infty$. So we need to prove that $H_{1}<\infty$ under the conditions of Theorem 2.2. For $p \geq 2$, noting that $\left\{A_{1}, A_{2}, \ldots, A_{n}\right\}$ is independent of $\left\{X_{n 1}, X_{n 2}, \ldots, X_{n n}\right\}$, similar to the proof of (3.5), one has by Lemma 1.1 that

$$
\begin{aligned}
H_{1}= & C_{1} \sum_{n=1}^{\infty} n^{\alpha p-2-q \alpha} E\left(\max _{1 \leq k \leq n}\left|\sum_{i=1}^{k}\left[A_{i} X_{n i}-E\left(A_{i} X_{n i} \mid g_{i-1}\right)\right]\right|^{q}\right) \\
\leq & C_{2} \sum_{n=1}^{\infty} n^{\alpha p-2-q \alpha} E\left(\sum_{i=1}^{n} E\left\{\left[A_{i} X_{n i}-E\left(A_{i} X_{n i} \mid g_{i-1}\right)\right]^{2} \mid g_{i-1}\right\}\right)^{q / 2} \\
& +C_{3} \sum_{n=1}^{\infty} n^{\alpha p-2-q \alpha} \sum_{i=1}^{n} E\left|A_{i} X_{n i}-E\left(A_{i} X_{n i} \mid g_{i-1}\right)\right|^{q} \\
= & C_{2} H_{11}+C_{3} H_{12} .
\end{aligned}
$$


Obviously, for $1 \leq i \leq n$, it has

$$
\begin{aligned}
& E\left\{\left[A_{i} X_{n i}-E\left(A_{i} X_{n i} \mid g_{i-1}\right)\right]^{2} \mid g_{i-1}\right\} \\
& \quad=E\left[A_{i}^{2} X_{i}^{2} I\left(\left|X_{i}\right| \leq n^{\alpha}\right) \mid g_{i-1}\right]-\left[E\left(A_{i} X_{i} I\left(\left|X_{i}\right| \leq n^{\alpha}\right) \mid g_{i-1}\right)\right]^{2} \\
& \quad \leq E\left[A_{i}^{2} X_{i}^{2} I\left(\left|X_{i}\right| \leq n^{\alpha}\right) \mid g_{i-1}\right] \leq E A_{i}^{2} E\left(X_{i}^{2} \mid g_{i-1}\right), \quad \text { a.s. }
\end{aligned}
$$

Combining (3.8) with $E\left[\sup _{i \geq 1} E\left(X_{i}^{2} \mid g_{i-1}\right)\right]^{q / 2}<\infty$, we obtain that

$$
\begin{aligned}
H_{11} & \leq \sum_{n=1}^{\infty} n^{\alpha p-2-q \alpha}\left(\sum_{i=1}^{n} E A_{i}^{2}\right)^{q / 2} E\left(\sup _{i \geq 1} E\left(X_{i}^{2} \mid g_{i-1}\right)\right)^{q / 2} \\
& \leq C_{4} \sum_{n=1}^{\infty} n^{\alpha p-2-q \alpha+q / 2}<\infty,
\end{aligned}
$$

following from the fact that $q>2(\alpha p-1) /(2 \alpha-1)$. Meanwhile, by $C_{r}$ inequality, Lemma 1.3 and (2.4),

$$
\begin{aligned}
H_{12} & \leq C_{5} \sum_{n=1}^{\infty} n^{\alpha p-2-q \alpha} \sum_{i=1}^{n} E\left|A_{i}\right|^{q} E\left[\left|X_{i}\right|^{q} I\left(\left|X_{i}\right| \leq n^{\alpha}\right)\right] \\
& \leq C_{6} \sum_{n=1}^{\infty} n^{\alpha p-1-q \alpha} E\left[X^{q} I\left(X \leq n^{\alpha}\right)\right]+C_{7} \sum_{n=1}^{\infty} n^{\alpha p-1} P\left(X>n^{\alpha}\right) \\
& \leq C_{6} \sum_{n=1}^{\infty} n^{\alpha p-1-q \alpha} E\left[X^{q} I\left(X \leq n^{\alpha}\right)\right]+C_{7} \sum_{n=1}^{\infty} n^{\alpha p-1-\alpha} E\left[X I\left(X>n^{\alpha}\right)\right] \\
& =: C_{6} H_{11}^{*}+C_{7} H_{12}^{*} .
\end{aligned}
$$

By the condition $p \geq 2$ and $\alpha>1 / 2$, we have that $2(\alpha p-1) /(2 \alpha-1)-p \geq 0$, which implies that $q>p$. So, one gets by $E X^{p}<\infty$ that

$$
\begin{aligned}
H_{11}^{*} & =\sum_{n=1}^{\infty} n^{\alpha p-1-q \alpha} \sum_{i=1}^{n} E\left[X^{q} I\left((i-1)^{\alpha}<X \leq i^{\alpha}\right)\right] \\
& =\sum_{i=1}^{\infty} E\left[X^{q} I\left((i-1)^{\alpha}<X \leq i^{\alpha}\right)\right] \sum_{n=i}^{\infty} n^{\alpha p-1-q \alpha} \\
& \leq C_{8} \sum_{i=1}^{\infty} E\left[X^{p} X^{q-p} I\left((i-1)^{\alpha}<X \leq i^{\alpha}\right)\right] i^{\alpha p-q \alpha} \leq C_{8} E X^{p}<\infty .
\end{aligned}
$$

By the proof of (3.3), it follows

$$
H_{12}^{*}=\sum_{n=1}^{\infty} n^{\alpha p-1-\alpha} E\left[X I\left(X>n^{\alpha}\right)\right] \leq C_{9} E X^{p}<\infty .
$$

Therefore, by (3.9)-(3.13), it has $H_{1}<\infty$. Consequently, it completes the proof of (2.2).

Finally, by the fact that $\alpha p>1$, similar to the proof of (3.4) in Yang et al. [10], it is easy to see that (2.3) holds for the case $\alpha p<2+\alpha$ and the case $\alpha p \geq 2+\alpha$. 
Proof of Theorem 2.3 Similar to the proof of Theorem 2.1, by Lemma 1.2, it can be checked that

$$
\begin{aligned}
\sum_{n=1}^{\infty} & n^{-2} E\left(\max _{1 \leq k \leq n}\left|\sum_{i=1}^{k} A_{i} X_{i}\right|-\varepsilon n^{\alpha}\right)^{+} \\
\leq & C_{1} \sum_{n=1}^{\infty} n^{-2-\alpha} E\left(\max _{1 \leq k \leq n}\left|\sum_{i=1}^{k}\left[A_{i} X_{n i}-E\left(A_{i} X_{n i} \mid g_{i-1}\right)\right]\right|^{2}\right) \\
& +\sum_{n=1}^{\infty} n^{-2} E\left(\max _{1 \leq k \leq n}\left|\sum_{i=1}^{k} A_{i} X_{i} I\left(\left|X_{i}\right|>n^{\alpha}\right)\right|\right) \\
& +\sum_{n=1}^{\infty} n^{-2} E\left(\max _{1 \leq k \leq n}\left|\sum_{i=1}^{k} E\left(A_{i} X_{n i} \mid g_{i-1}\right)\right|\right) \\
:= & J_{1}+J_{2}+J_{3} .
\end{aligned}
$$

Similarly to the proof of (3.3), we have

$$
\begin{aligned}
J_{2} & \leq C_{1} \sum_{n=1}^{\infty} n^{-1} E\left[X I\left(X>n^{\alpha}\right)\right] \\
& =C_{1} \sum_{n=1}^{\infty} n^{-1} \sum_{m=n}^{\infty} E\left[X I\left(m^{\alpha}<X \leq(m+1)^{\alpha}\right)\right] \\
& =C_{1} \sum_{m=1}^{\infty} E\left[X I\left(m^{\alpha}<X \leq(m+1)^{\alpha}\right)\right] \sum_{n=1}^{m} n^{-1} \\
& \leq C_{2} \sum_{m=1}^{\infty} \ln (1+m) E\left[X I\left(m^{\alpha}<X \leq(m+1)^{\alpha}\right)\right] \\
& \leq C_{3} E[X \ln (1+X)]<\infty .
\end{aligned}
$$

Meanwhile, by the proofs of (3.4) and (3.15), we get

$$
J_{3} \leq C_{1} \sum_{n=1}^{\infty} n^{-1} E\left[X I\left(X>n^{\alpha}\right)\right] \leq C_{2} E[X \ln (1+X)]<\infty \text {. }
$$

On the other hand, by the proof of (3.5), it can be checked that for $\alpha>0$,

$$
\begin{aligned}
J_{1} & \leq C_{2} \sum_{n=1}^{\infty} n^{-2-\alpha} \sum_{i=1}^{n} E\left(A_{i} X_{n i}\right)^{2}=C_{2} \sum_{n=1}^{\infty} n^{-2-\alpha} \sum_{i=1}^{n} E A_{i}^{2} E X_{n i}^{2} \\
& \leq C_{3} \sum_{n=1}^{\infty} n^{-1-\alpha} E\left[X^{2} I\left(X \leq n^{\alpha}\right)\right]+C_{4} \sum_{n=1}^{\infty} n^{\alpha-1} P\left(X>n^{\alpha}\right) \\
& \leq C_{3} \sum_{n=1}^{\infty} n^{-1-\alpha} \sum_{i=1}^{n} E\left[X^{2} I\left((i-1)^{\alpha}<X \leq i^{\alpha}\right)\right]+C_{4} \sum_{n=1}^{\infty} n^{-1} E\left[X I\left(X>n^{\alpha}\right)\right] \\
& \leq C_{3} \sum_{i=1}^{\infty} E\left[X^{2} I\left((i-1)^{\alpha}<X \leq i^{\alpha}\right)\right] \sum_{n=i}^{\infty} n^{-1-\alpha}+C_{5} E[X \ln (1+X)]
\end{aligned}
$$




$$
\begin{aligned}
& \leq C_{4} \sum_{i=1}^{\infty} E\left[X^{2} I\left((i-1)^{\alpha}<X \leq i^{\alpha}\right)\right] i^{-\alpha}+C_{5} E[X \ln (1+X)] \\
& \leq C_{6} E X+C_{5} E[X \ln (1+X)]<\infty
\end{aligned}
$$

Therefore, by (3.14)-(3.17), one gets (2.5) immediately. Similar to the proof of (2.3), it is easy to have (2.6). Obviously, by the proof of (2.11) in Remark 2.2, (2.7) also holds under the conditions of Theorem 2.3. Finally, by the proof of Theorem 12.1 of Gut [11] and the proof of (3.2) in Yang et al. [10], for $\alpha>1$, it is easy to get (2.8).

Proof of Theorem 2.4 For $n \geq 1$, we also denote $X_{n i}=X_{i} I\left(\left|X_{i}\right| \leq n^{\alpha}\right), 1 \leq i \leq n$. It is easy to see that

$$
P\left(\max _{1 \leq k \leq n}\left|\sum_{i=1}^{k} A_{i} X_{i}\right|>\varepsilon n^{\alpha}\right) \leq \sum_{i=1}^{n} P\left(\left|X_{i}\right|>n^{\alpha}\right)+P\left(\max _{1 \leq k \leq n}\left|\sum_{i=1}^{k} A_{i} X_{n i}\right|>\varepsilon n^{\alpha}\right) .
$$

For the case of $\alpha=1$, there exists a $\delta>0$ such that $\lim _{n \rightarrow \infty} \frac{\max _{1 \leq i \leq n} E\left[\left|X_{i}\right|^{1+\delta} \mid g_{i-1}\right]}{n^{\delta}}=0$, a.s. So by $E\left(A_{n} X_{n} \mid g_{n-1}\right)=0$, a.s., $n \geq 1$, we can check that

$$
\begin{aligned}
\frac{1}{n^{\alpha}}\left(\max _{1 \leq k \leq n}\left|\sum_{i=1}^{k} E\left(A_{i} X_{n i} \mid g_{i-1}\right)\right|\right) & =\frac{1}{n}\left(\max _{1 \leq k \leq n}\left|\sum_{i=1}^{k} E\left[A_{i} X_{i} I\left(\left|X_{i}\right| \leq n\right) \mid g_{i-1}\right]\right|\right) \\
& =\frac{1}{n}\left(\max _{1 \leq k \leq n}\left|\sum_{i=1}^{k} E\left[A_{i} X_{i} I\left(\left|X_{i}\right|>n\right) \mid g_{i-1}\right]\right|\right) \\
& \leq \frac{1}{n} \sum_{i=1}^{n} E\left|A_{i}\right| E\left[\left|X_{i}\right| I\left(\left|X_{i}\right|>n\right) \mid g_{i-1}\right] \\
& \leq \frac{1}{n^{1+\delta}} \sum_{i=1}^{n} E\left|A_{i}\right| E\left[\left|X_{i}\right|^{1+\delta} \mid g_{i-1}\right] \\
& \leq \frac{K}{n^{\delta}} \max _{1 \leq i \leq n} E\left[\left|X_{i}\right|^{1+\delta} \mid g_{i-1}\right] \rightarrow 0, \quad \text { a.s., }
\end{aligned}
$$

as $n \rightarrow \infty$.

Otherwise, for the case of $\alpha>1$, it is assumed that $\lim _{n \rightarrow \infty} \frac{\max _{1 \leq i \leq n} E\left[\mid X_{i} \| g_{i-1}\right]}{n^{\lambda}}=0$, a.s., for any $\lambda>0$. Consequently, for any $\alpha>1$, it follows that

$$
\begin{aligned}
\frac{1}{n^{\alpha}}\left(\max _{1 \leq k \leq n}\left|\sum_{i=1}^{k} E\left(A_{i} X_{n i} \mid g_{i-1}\right)\right|\right) & =\frac{1}{n^{\alpha}}\left(\max _{1 \leq k \leq n}\left|\sum_{i=1}^{k} E\left[A_{i} X_{i} I\left(\left|X_{i}\right| \leq n\right) \mid g_{i-1}\right]\right|\right) \\
& \leq \frac{1}{n^{\alpha}} \sum_{i=1}^{n} E\left|A_{i}\right| E\left[\left|X_{i}\right| I\left(\left|X_{i}\right| \leq n\right) \mid g_{i-1}\right] \\
& \leq \frac{K_{1}}{n^{\alpha-1}} \max _{1 \leq i \leq n} E\left[\left|X_{i}\right| \mid g_{i-1}\right] \rightarrow 0, \quad \text { a.s., }
\end{aligned}
$$

as $n \rightarrow \infty$. Meanwhile,

$$
\sum_{n=1}^{\infty} n^{\alpha-2} \sum_{i=1}^{n} P\left(\left|X_{i}\right|>n^{\alpha}\right) \leq K_{1} \sum_{n=1}^{\infty} n^{\alpha-1} P\left(X>n^{\alpha}\right) \leq K_{2} E X<\infty .
$$


By (3.18) and (3.19), to prove (2.7), it suffices to show that

$$
I_{3}=\sum_{n=1}^{\infty} n^{\alpha-2} P\left(\max _{1 \leq k \leq n}\left|\sum_{i=1}^{k}\left[A_{i} X_{n i}-E\left(A_{i} X_{n i} \mid \mathscr{G}_{i-1}\right)\right]\right|>\frac{\varepsilon n^{\alpha}}{2}\right)<\infty .
$$

Obviously, by Markov's inequality and the proofs of (3.5), (3.6), (3.19), one can check that

$$
\begin{aligned}
I_{3} & \leq \frac{4}{\varepsilon^{2}} \sum_{n=1}^{\infty} n^{-2-\alpha} E\left(\max _{1 \leq k \leq n}\left|\sum_{i=1}^{k}\left[A_{i} X_{n i}-E\left(A_{i} X_{n i} \mid g_{i-1}\right)\right]\right|^{2}\right) \\
& \leq K_{1} \sum_{n=1}^{\infty} n^{-1-\alpha} E\left[X^{2} I\left(X \leq n^{\alpha}\right)\right]+K_{2} \sum_{n=1}^{\infty} n^{\alpha-1} P\left(X>n^{\alpha}\right) \\
& \leq K_{3} E X<\infty .
\end{aligned}
$$

On the other hand, by proof of Theorem 12.1 of Gut [11] and the proof of (3.2) in Yang et al. [10], we can easily obtain (2.8) for $\alpha>1$.

\section{Competing interests}

The authors declare that they have no competing interests.

\section{Authors' contributions}

All authors read and approved the final manuscript.

\section{Author details}

'School of Mathematical Science, Anhui University, Hefei, China. ${ }^{2}$ Department of System Engineering and Engineering Management, The Chinese University of Hong Kong, Hong Kong, China.

\section{Acknowledgements}

The authors are most grateful to editor prof. Soo Hak Sung and two anonymous referees for their careful reading and insightful comments, which helped to significantly improve an earlier version of this paper. Supported by the NNSF of China (11171001, 11201001), Natural Science Foundation of Anhui Province (1208085QA03, 1308085QA03), Talents Youth Fund of Anhui Province Universities (2012SQRL204) and Doctoral Research Start-up Funds Projects of Anhui University.

Received: 31 March 2013 Accepted: 6 August 2013 Published: 21 August 2013

\section{References}

1. Hsu, PL, Robbins, H: Complete convergence and the law of large numbers. Proc. Natl. Acad. Sci. USA 33(2), 25-31 (1947)

2. Erdös, P: On a theorem of Hsu and Robbins. Ann. Math. Stat. 20(2), 286-291 (1949)

3. Baum, LE, Katz, M: Convergence rates in the law of large numbers. Trans. Am. Math. Soc. 120(1), 108-123 (1965)

4. Shao, QM: A moment inequality and its application. Acta Math. Sin. 31A(6), 736-747 (1988)

5. Yu, KF: Complete convergence of weighted sums of martingale differences. J. Theor. Probab. 3(2), 339-347 (1990)

6. Ghosal, S, Chandra, TK: Complete convergence of martingale arrays. J. Theor. Probab. 11(3), 621-631 (1998)

7. Stoica, G: Baum-Katz-Nagaev type results for martingales. J. Math. Anal. Appl. 336(2), 1489-1492 (2007)

8. Stoica, G: A note on the rate of convergence in the strong law of large numbers for martingales. J. Math. Anal. Appl. 381(2), 910-913 (2011)

9. Wang, $\mathrm{XJ}, \mathrm{Hu}, \mathrm{SH}$, Yang, WZ, Wang, $\mathrm{XH}$ : Convergence rates in the strong law of large numbers for martingale difference sequences. Abstr. Appl. Anal. 2012, Article ID 572493 (2012)

10. Yang, WZ, Hu, SH, Wang, XJ: Complete convergence for moving average process of martingale differences. Discrete Dyn. Nat. Soc. 2012, Article ID 128492 (2012)

11. Gut, A: Probability: A Graduate Course. Springer, New York (2005)

12. Chen, PY, Hu, T-C, Volodin, A: Limiting behaviour of moving average processes under $\varphi$-mixing assumption. Stat. Probab. Lett. 79(1), 105-111 (2009)

13. Sung, SH: Moment inequalities and complete moment convergence. J. Inequal. Appl. 2009, Article ID 271265 (2009)

14. Sung, SH: Complete convergence for weighted sums of $\rho^{*}$-mixing random variables. Discrete Dyn. Nat. Soc. 2010, Article ID 630608 (2010)

15. Sung, $\mathrm{SH}$ : Convergence of moving average processes for dependent random variables. Commun. Stat., Theory Methods 40(13), 2366-2376 (2011)

16. Sung, $\mathrm{SH}$ : Complete qth moment convergence for arrays of random variables. J. Inequal. Appl. 2013, Article ID 24 (2013) 
17. Sung, SH, Volodin, A: A note on the rate of complete convergence for weighted sums of arrays of Banach space valued random elements. Stoch. Anal. Appl. 29(2), 282-291 (2011)

18. Hu, T-C, Rosalsky, A, Volodin, A: A complete convergence theorem for row sums from arrays of rowwise independent random elements in Rademacher type p Banach spaces. Stoch. Anal. Appl. 30(2), 343-353 (2012)

19. Thanh, LV, Yin, G: Almost sure and complete convergence of randomly weighted sums of independent random elements in Banach spaces. Taiwan. J. Math. 15(4), 1759-1781 (2011)

20. Cabrera, MO, Rosalsky, A, Volodin, A: Some theorems on conditional mean convergence and conditional almost sure convergence for randomly weighted sums of dependent random variables. Test 21(2), 369-385 (2012)

21. Hall, P, Heyde, CC: Martingale Limit Theory and Its Application. Academic Press, New York (1980)

22. Huang, DW, Guo, L: Estimation of nonstationary ARMAX models based on the Hannan-Rissanen method. Ann. Stat. 18(4), 1729-1756 (1990)

23. Thanh, LV, Yin, G, Wang, LY: State observers with random sampling times and convergence analysis of double-indexed and randomly weighted sums of mixing processes. SIAM J. Control Optim. 49(1), 106-124 (2011)

doi:10.1186/1029-242X-2013-396

Cite this article as: Yang et al.: Complete moment convergence for randomly weighted sums of martingale

differences. Journal of Inequalities and Applications 2013 2013:396.

\section{Submit your manuscript to a SpringerOpen ${ }^{\ominus}$ journal and benefit from:}

- Convenient online submission

- Rigorous peer review

- Immediate publication on acceptance

- Open access: articles freely available online

- High visibility within the field

- Retaining the copyright to your article 